\title{
Estudio de la sensibilidad a la velocidad de deformación en régimen superplástico del inconel 718 mediante ensayos con perturbación cíclica de la velocidad de deformación
}

\author{
M. URDANPILLETA*, C. ZUBILLAGA, J. GIL
}

CEIT (Ctro. de Estudios e Investigaciones Técnicas de Guipúzcoa) y TECNUN (Campus Tecnológico, Universidad de Navarra) 20018 San Sebastián (España)

(*) Dirección actual: Dpto. Ciencias Básicas, Escuela Politécnica Superior, Universidad de Mondragón, 20500 Mondragón

\begin{abstract}
La imposición de una perturbación sinusoidal de frecuencia variable a la velocidad de deformación de un material sometido a tracción monótona es una posible técnica de estudio de la cinética de la deformación plástica apenas explorada. La aplicación de esta técnica a un Inconel 718 en el régimen superplástico ha permitido obtener el valor de la sensibilidad a la velocidad de deformación instantánea, m, de forma continua con la deformación. Esto permite una caracterización más completa que con las técnicas convencionales, en las que la sensibilidad $\mathrm{m}$ se determina a partir de ensayos en los que se cambia de forma nominalmente instantánea la velocidad de deformación. En efecto, la técnica propuesta permite poner de manifiesto la influencia de la derivada temporal de la velocidad de deformación, $\ddot{\varepsilon}$, sobre el valor de m. Para la comparación de ambos tipos de medidas, se han realizado ensayos tanto con perturbación sinusoidal como con cambio instantáneo de velocidad de deformación. Se ha estudiado el rango de temperaturas entre $925^{\circ} \mathrm{C}$ y $1000^{\circ} \mathrm{C}$ y de velocidades entre $10^{-4} \mathrm{~s}^{-1}$ y $10^{-2}$ $\mathrm{s}^{-1}$. Se ha analizado asimismo la respuesta dinámica del sistema, en términos de amplitud y desfase de la señal de tensión resultante respecto de la perturbación sinusoidal de velocidad de deformación impuesta.
\end{abstract}

Palabras clave: Superplasticidad, Inconel 718, sensibilidad a la velocidad de deformación, anelasticidad.

\section{Study of the superplasticity of inconel 718 using tests with cyclic perturbation of strain rate}

The imposition of a sinusoidal perturbation of variable frequency to the strain rate of a material subjected to a monotonic tensile test is an easy technique to study the kinetics of plastic deformation that has been scarcely used. The application of this technique to an Inconel 718 in its superplastic regime allows for obtaining has permitted to obtain the value of the instantaneous strain rate sensitivity, $\mathrm{m}$, in a continuous way as deformation. It is then possible a more complete characterization than that offered by conventional techniques, where strain rate sensitivity is obtained from the response to several instantaneous strain rate changes. The proposed method has revealed the influence that the time derivative of strain rate, $\ddot{\varepsilon}$, has on the value of $\mathrm{m}$. For a comparison of the rexults of both types of measurement, tests with sinusoidal perturbation and with instantaneous strain rate changes have been performed. The temperature and strain rate ranges studied span from $925^{\circ} \mathrm{C}$ to $1000^{\circ} \mathrm{C}$ and from $10^{-4} \mathrm{~s}^{-1}$ to $10^{-2} \mathrm{~s}^{-1}$. The dynamic response of the system has been analyzed too in terms of amplitude and phase shift of the resulting stress signal response to the imposed sinusoidal perturbation of strain rate.

Keywords: Superplasticity, Inconel 718, strain rate sensitivity, anelasticity.

\section{INTRODUCCIÓN}

Se han utilizado hasta la fecha varios métodos posibles para la determinación de la sensibilidad a la velocidad de deformación, m, en los materiales superplásticos. Los ensayos empleados pueden ser de tensión, de compresión, de relajación, etc (1). Todos ellos implican cambios bruscos en la velocidad o en la tensión, lo cual no permite obtener $m$ de forma continua con la deformación. La técnica aquí propuesta para el estudio de la superplasticidad (que sólo ha sido empleada con anterioridad por Ling et al (2) para el estudio del envejecimiento dinámico), permite esto último, al consistir en cambios suaves y continuos en la velocidad de deformación, de los que se puede obtener $\mathrm{m}$ a partir de la derivada instantánea de la tensión respecto de la velocidad de deformación. Además, se obtiene la dependencia de $\mathrm{m}$ con la derivada temporal de la velocidad de deformación (o "aceleración de deformación"), lo cual puede ser de gran utilidad en procesos industriales en los que la velocidad de deformación no tiene por qué ser constante. En concreto, se han impuesto, sobre una velocidad de deformación constante tractiva de base, perturbaciones sinusoidales de frecuencia variable y amplitud mitad de la velocidad de deformación de base. Estos ensayos se han comparado con otra serie de ensayos convencionales (denominados de tipo escalón) en los que, a una velocidad de deformación de base constante, se le añaden excursiones pequeñas y espaciadas a una segunda velocidad de deformación $50 \%$ superior a la de base.

La sensibilidad a la velocidad de deformación medida mediante cambios instantáneos de velocidad de deformación proporciona un dato a estructura constante (la del instante del cambio). Si se trata de caracterizar un proceso en que la deformación plástica está induciendo cambios estructurales, en los ensayos de variación continua de velocidad de deformación sería necesario tener en cuenta el cambio estructural en el análisis de la sensibilidad a la velocidad de deformación. Esto no es relevante en el régimen superplástico, en el que los cambios estructurales son mínimos.

\section{PARTE EXPERIMENTAL}

El material de recepción, proporcionado por ITP, es una chapa laminada de Inconel 718 de $1 \mathrm{~mm}$ de grosor, de calidad SPF (para conformado superplástico), con un tamaño de grano de $5.0 \pm 0.3$ (medido por el promedio de la intersección lineal media en las tres direcciones 
de la chapa). Las probetas, de $30 \mathrm{~mm}$ de zona recta, fueron ensayadas a tracción mediante una máquina Instron 8562 controlada por ordenador. Este control permite la obtención de cualesquiera señales de velocidad de deformación verdadera con gran precisión (siempre dentro de un rango de velocidades y cargas como el estudiado en este trabajo). Los efectos de rigidez de la máquina fueron despreciados debido a las bajas cargas y velocidades empleadas.

Los ensayos convencionales de cambio brusco en la velocidad de deformación, a los que se denominará como ensayos "de escalón" fueros realizados a las temperaturas de $925^{\circ}, 937.5^{\circ}, 950^{\circ}, 962.5^{\circ}, 975^{\circ}$,

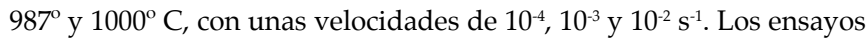
de perturbación cíclica exploraron la región de $925^{\circ}, 950^{\circ}, 975^{\circ}$ y $1000^{\circ}$ C, con una velocidad de deformación de base de $10^{-3} \mathrm{~s}^{-1} \mathrm{y}$ con unos periodos de oscilación de 300, 200, 150, 100, 75, 50, 37.5, 25 y $18.75 \mathrm{~s}$.

Una descripción detallada de las técnicas experimentales utilizadas y de los resultados obtenidos se expone en la ref. (3).

\section{RESULTADOS}

\subsection{Ensayos de tipo escalón}

Los resultados de sensibilidad a la velocidad de deformación obtenidos por el método de cambio nominalmente instantáneo, con una velocidad de deformación de base de $10^{-3} \mathrm{~s}^{-1}$, se muestran en la figura 1. El incremento de tensión se obtuvo extrapolando hacia atrás la nueva curva de endurecimiento, para eliminar el transitorio que se observa en la deformación inmediata al cambio. Los valores obtenidos dependen débilmente de la temperatura, y rondan $\mathrm{m}=0.2-0.3$, con un máximo entre 950 y $975^{\circ} \mathrm{C}$.



Figura 1. Sensibilidad a la velocidad de deformación para ensayos tipo escalón con velocidad de deformación de base de $10^{-3} \mathrm{~s}^{-1}$

\subsection{Ensayos con perturbación cíclica en la velocidad de de- formación}

\subsubsection{SENSIBILIDAD A LA VELOCIDAD DE DEFORMACIÓN}

Para obtener los valores de sensibilidad a la velocidad de deformación mediante esta técnica se ha utilizado la ecuación

$$
\frac{d \sigma}{d t}=\left.\frac{\partial \sigma}{\partial t}\right|_{\varepsilon, \dot{\varepsilon}}+\left.\frac{\partial \sigma}{\partial \varepsilon}\right|_{\dot{\varepsilon}} \cdot \dot{\varepsilon}+\left.\frac{\partial \sigma}{\partial \varepsilon}\right|_{\varepsilon} \cdot \ddot{\varepsilon}
$$

donde se han despreciado los siguientes términos de la serie y donde la tercera derivada parcial es igual a la sensibilidad $\mathrm{m}$. El término dependiente explícitamente del tiempo, $\left.\frac{\partial \sigma}{\partial t}\right|_{\varepsilon, \dot{\varepsilon}}$, da cuenta de la relajación del sistema y es despreciable para tiempos de ensayo largos, como los utilizados. Como puede comprobarse, considerando únicamente las partes elástica y plástica de la deformación, la integración de la ecuación

$$
\dot{\varepsilon}=\dot{\varepsilon}_{E l}+\dot{\varepsilon}_{P l}=\frac{\sigma}{E}+A \sigma^{n}=0
$$

(donde $E$ es el módulo de Young) conduce a un valor despreciable de $\partial \sigma$

$\left.\frac{\partial \sigma}{\partial t}\right|_{\varepsilon, \dot{\varepsilon}}$ para los valores $E$ y $A$ considerados (respectivamente, 200 GPa y $10^{-13} \mathrm{~s}^{-1} \mathrm{MPa}^{-5}$, con $\sigma \approx 100 \mathrm{MPa}$ y $\mathrm{n}=5$ ).

Los valores de $m$ medidos por esta técnica dependen débilmente de la frecuencia de la perturbación (figura 2) y coinciden aceptablemente con los obtenidos mediante los ensayos de cambio instantáneo de velocidad de deformación.

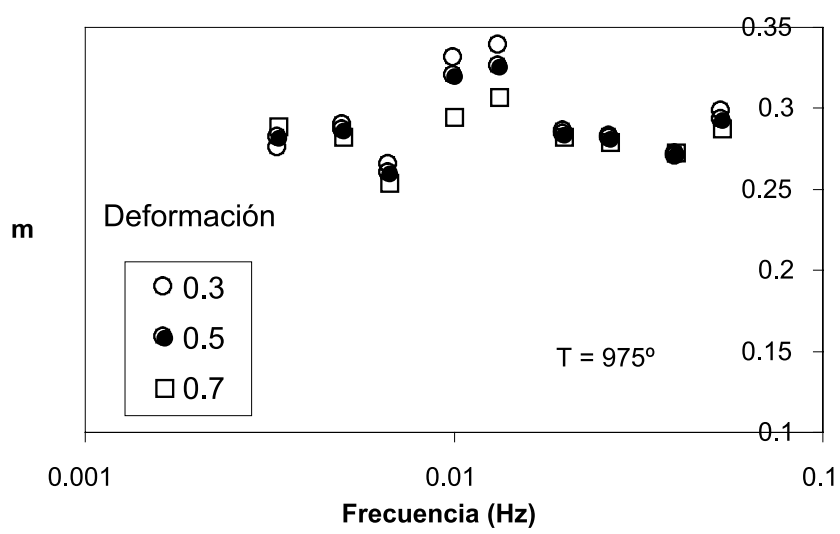

Figura 2. Sensibilidad a la velocidad de deformación para ensayos de perturbación cíclica a $975^{\circ} \mathrm{C}$ y velocidad de deformación de base de $10^{-3} \mathrm{~s}^{-1}$

\subsubsection{EFECTO DE LA ACELERACIÓN DE DEFORMACIÓN SOBRE LA SENSIBILIDAD M}

Considerando una ecuación potencial en la que el exponente m dependiera de la aceleración de deformación

$$
\sigma=F(\ddot{\varepsilon}) \dot{\varepsilon}^{m(\ddot{\varepsilon})}
$$

tomando logaritmos puede obtenerse $\mathrm{m}(\ddot{\varepsilon})$ a partir de un ajuste lineal. Los valores así obtenidos se muestran en la figura 3 para la temperatura de $950^{\circ} \mathrm{C}$. La dependencia con la aceleración de deformación es débil, pero apreciable: se observa una ligera subida conforme aumenta la aceleración. Es decir, para aceleraciones negativas (que coinciden con bajadas de la velocidad de deformación) la sensibilidad es ligeramente menor que para aceleraciones positivas (que corresponden a subidas en la velocidad de deformación). El resultado es similar para el resto de temperaturas ensayadas.

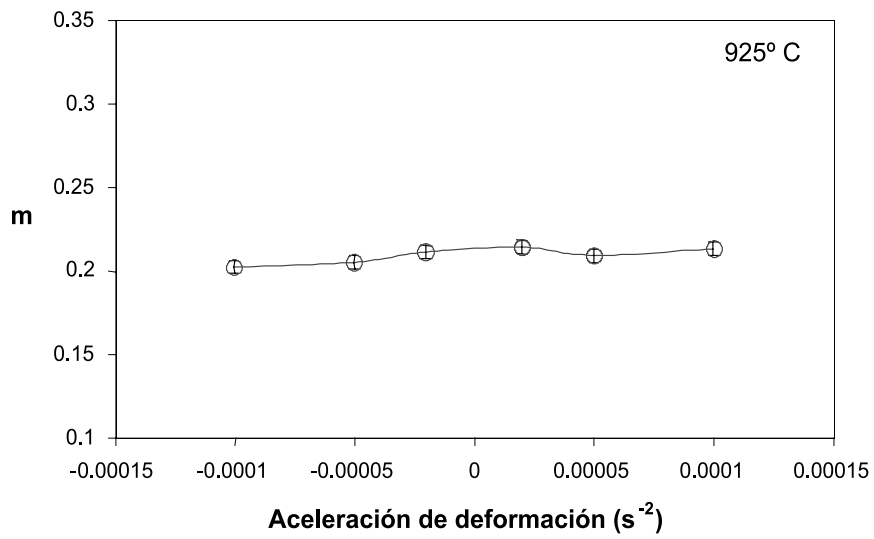

Figura 3. Dependencia de $\mathrm{m}$ con la velocidad de deformación. Ensayos a $925^{\circ} \mathrm{C}$ 


\subsubsection{RESPUESTA EN TENSIÓN EN TÉRMINOS DE AMPLI- TUD Y DESFASE}

Si se tiene en cuenta que la señal perturbada de tensión tiene la forma

$$
\sigma=\sigma_{0}(t)+\sigma_{1} \cos (\omega t+\delta)
$$

puede ajustarse la señal de velocidad de deformación de respuesta a una función

$$
\dot{\varepsilon}=\dot{\varepsilon}_{0}+\dot{\varepsilon}_{1} \cos \omega t=\dot{\varepsilon}_{0}\left(1+\frac{1}{2} \cos \omega t\right)
$$

y estudiar dicha respuesta en términos de amplitud $\sigma_{1}$ y desfase $\delta$ respecto de la señal de entrada.

Las gráficas de desfase en función de la frecuencia se muestran en la figura 4 para las cuatro temperaturas estudiadas. Se observan dos tipos de comportamiento: a las temperaturas de $925^{\circ} \mathrm{C}$ y $950^{\circ} \mathrm{C}$ coexisten desfases positivos y negativos, mientras que para $1000^{\circ} \mathrm{C}$ sólo existen valores negativos. El valor absoluto de los desfases negativos aumenta al crecer la frecuencia de la oscilación de la velocidad.

\section{DISCUSIÓN}

Las diferencias en comportamiento entre las temperaturas superiores e inferiores a $975^{\circ} \mathrm{C}$ (en concreto el descenso del valor de la sensibilidad en los ensayos de escalón a $10^{-4} \mathrm{~s}^{-1}$ y la ya mencionada forma de los desfases) pueden achacarse al comienzo de la disolución, hacia los $975^{\circ} \mathrm{C}$, de la fase $\delta\left(\mathrm{Ni}_{3} \mathrm{Nb}\right)$ presente en las fronteras de grano. Esta fase actúa anclando las fronteras e impidiendo el crecimiento del grano, manteniendo las propiedades superplásticas del material. Sin embargo, se ha apuntado en la bibliografía (4) que las partículas de fase $\delta$ pueden actuar también inhibiendo el deslizamiento de fronteras de grano, al presentar obstáculos en los que se concentran tensiones. Estas tensiones, al ser relajadas por difusión o por movimiento de dislocaciones, pasarían a concentrarse en los puntos triples (5). Esta posible inhibición del deslizamiento de fronteras de grano, junto con los bajos valores de sensibilidad a la velocidad de deformación encontrados, dan pie a afirmar que la contribución del movimiento de dislocaciones desempeña un importante papel en la deformación del Inconel 718 en las condiciones estudiadas. En efecto, los valores de $\mathrm{m}$, por debajo de 0.4 , son típicos de la denominada región III de los materiales superplásticos, en los que se considera que el mecanismo dominante de la cinética observada es la fluencia de dislocaciones. Además, es razonable pensar que la influencia de la deformación por dislocaciones es aún mayor en la región de temperaturas por encima de $975^{\circ} \mathrm{C}$ debido al crecimiento del grano (comprobado metalográficamente).

Finalmente, la influencia de la frecuencia en los desfases de respuesta observados o la influencia de la aceleración de la deformación sobre la sensibilidad de la tensión a la velocidad de deformación pueden explicarse por una contribución anelástica a la deformación durante las oscilaciones de velocidad. Esta contribución es relevante en materiales superplásticos, como se señala en los trabajos de Schneibel y Hazzledine (6) acerca de un $\mathrm{Pb}$-Sn eutéctico superplástico.

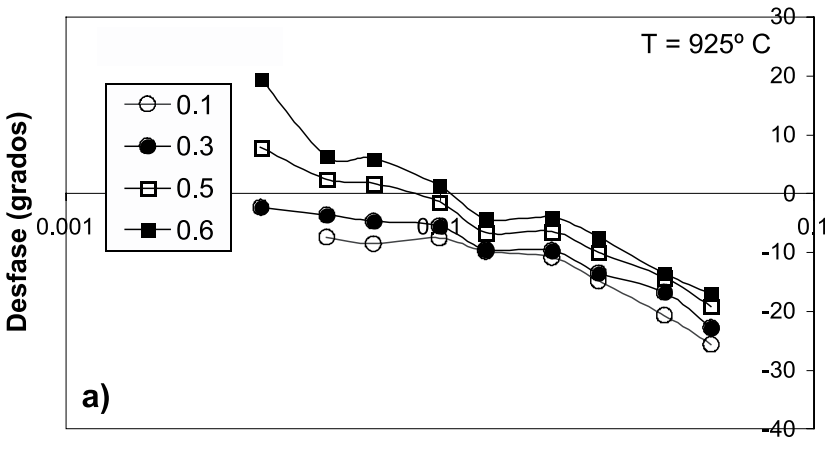

Frecuencia (Hz)

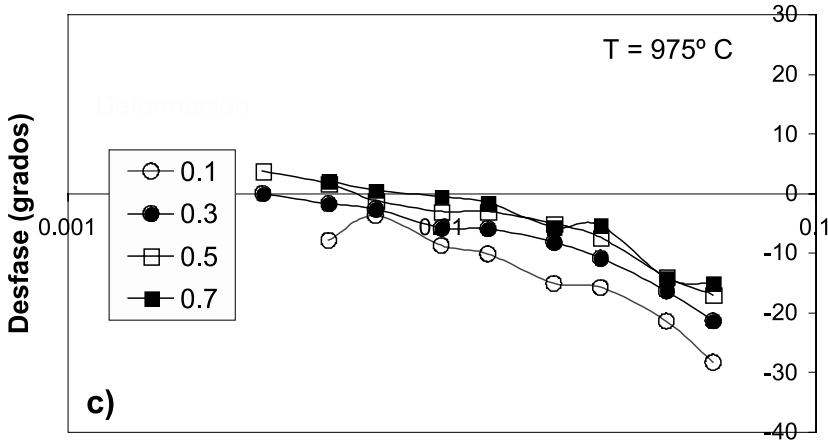

Frecuencia $(\mathrm{Hz})$



Frecuencia $(\mathrm{Hz})$

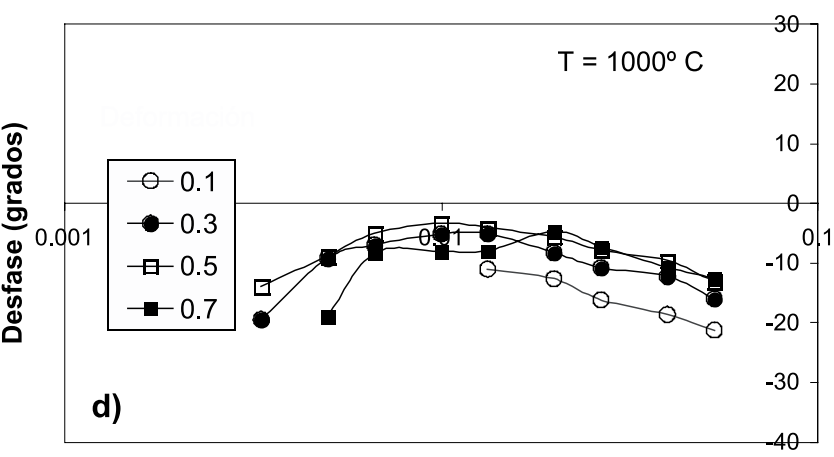

Frecuencia $(\mathrm{Hz})$

Figura 4. Desfases entre la velocidad de deformación impuesta y la tensión para ensayos a a) 925, b) $950^{\circ}$ C, c) $975^{\circ} \mathrm{C}$ y d) $1000^{\circ} \mathrm{C}$. Todos para velocidad de deformación de base de $10^{-3} \mathrm{~s}^{-1}$. 


\section{CONCLUSIONES}

Los resultados obtenidos permiten extraer las siguientes conclusiones:

(1) Se ha observado la aparición de dos regímenes de comportamiento diferenciados, por encima y por debajo de $975^{\circ} \mathrm{C}$, con un comportamiento superplástico óptimo entre 950 y $975^{\circ} \mathrm{C}$. Por encima de $975^{\circ} \mathrm{C}$ la sensibilidad a la velocidad de deformación, m, decrece por debajo de 0.2 .

(2) La transición de comportamiento puede atribuirse a la evolución microestructural del material: a $975^{\circ} \mathrm{C}$, la disolución de la fase $\delta$ presente permite un fuerte crecimiento del tamaño de grano, que afecta a las propiedades mecánicas del material.

(3) Los valores de sensibilidad obtenidos por los dos métodos de ensayos de escalón y de perturbación cíclica en la velocidad de deformación son muy similares.

(4) La sensibilidad a la velocidad de deformación es ligeramente mayor para aceleraciones de deformación positivas comparadas con la medida para aceleraciones negativas.

\section{AGRADECIMIENTOS}

Los autores quieren agradecer a la Industria de Turbopropulsores (ITP, Zamudio, España) el suministro del material y al Gobierno Vasco por la financiación parcial del trabajo realizado (proyecto en cooperación DT-ITP-98-001).

\section{BIBLIOGRAFÍA}

1. M. Yoshizawa, H. Ohsawa. "Estimation of strain rate sensitivity in superplastic compression tests", pp. 260-267 en "Superplasticity: 60 years after Pearson". Ed. N. Ridley. The Institute of Materials, London, (UK), 1995.

2. C.P. Ling, Y. Estrin, P.G. McCormick. "Ramped oscillatory testing: a new technique to examine dynamic strain ageing", Acta mater. 42, 5, pp. 15411549 (1994).

3. M. Urdanpilleta. "Deformación superplástica de las aleaciones Inconel 718SPF y Ti-6\% Al-4\% V". Tesis de Doctorado, TECNUN, Universidad de Navarra, San Sebastián, 2003.

4. W. Chen, M.C. Chaturdevi, "The effect of grain boundary precipitates on the creep behavior of Inconel 718", Mat. Sci. Eng. A, 183, pp. 81-89 (1994).

5. W. Chen, M.C. Chaturdevi, "Dependence of creep fracture of Inconel 718", Acta mater. vol. 45, 7, pp. 2735-2746 (1997).

6. J.H. Schneibel, P.M. Hazzledine, "Superanelasticity in Sn-Pb alloys", Acta metall., 30, pp. 1223-1230 (1982).

Recibido: 1.2 .03

Aceptado: 30.11 .03 